and no transmissible antibiotic resistance was detected. It has inherent acid resistance and is able to tolerate $\mathrm{pH} 2$ with a survival rate of $20 \%$, increasing to $76 \%$ at $\mathrm{pH} 3$. It is able to metabolise a range of carbohydrates, including lactose, glucose, sucrose, fructose, mannose, ribose, raffinose and $\mathrm{N}$ acetyl glucosamine.

Conclusion Our candidate probiotic, S thermophilus NCIMB 41856 has properties that would enable it to survive in and colonise the human intestine. It can survive gastric levels of acidity, utilise a range of carbohydrates present in the intestine, and is able to bind to epithelial cells. It is also able to prevent the binding of potential pathogens. Furthermore, it does not possess undesirable antibiotic resistance or produce harmful metabolic products. These features indicate that it would be safe and potentially effective in use as a probiotic. We propose to test this in vivo in future work.

Competing interests None declared.

\section{PWE-231 MRI IS CORRELATED TO FAECAL CALPROTECTIN LEVEL IN THE EVALUATION OF SMALL BOWEL AND COLONIC CROHN'S DISEASE}

doi:10.1136/gutjnl-2012-302514d.231

\begin{abstract}
${ }^{1} \mathrm{~J}$ Makanyanga, ${ }^{2} \mathrm{D}$ Pendse, ${ }^{2} \mathrm{E}$ Atkins, ${ }^{1} \mathrm{~A}$ Menys, ${ }^{3} \mathrm{~S}$ Bloom, ${ }^{3} \mathrm{~S}$ McCartney, ${ }^{2} \mathrm{~S}$ Punwani, ${ }^{2} \mathrm{~S}$ Halligan, ${ }^{2} \mathrm{~S}$ Taylor. ${ }^{1}$ Centre for Medical Imaging, University College London, UK; ${ }^{2}$ Department of Specialist Radiology, University College London Hospitals, London, UK; ${ }^{3}$ Department of Gastroenterology, University College London Hospitals, London, UK
\end{abstract}

Introduction Crohn's disease management requires knowledge of overall disease burden. A new MRI score of Crohn's disease activity was tested against reference standards of global activity-Harvey Bradshaw index (HBI) and faecal calprotectin.

Methods 34 patients (15 male) median age 33 (range 17-78) with known or suspected Crohn's disease underwent MR enterography (axial/coronal HASTE, TrueFisp and post gadolinium coronal VIBE/ THRIVE at $1.5 \mathrm{~T}(\mathrm{n}=24)$ or $3 \mathrm{~T}(\mathrm{n}=10))$. Same day HBI questionnaire and faecal calprotectin were measured. Two observers qualitatively graded bowel wall thickness, mural T2 signal, mesenteric oedema, T1 enhancement and colonic haustral loss from 0 (normal) to 3 (most abnormal) for the jejunum, proximal ileum, terminal ileum and colon (six segments). Each individual small bowel and colonic segmental score was multiplied according to the length of disease in that segment ( $0-5 \mathrm{~cm} \times 1,6-15 \mathrm{~cm} \times 1.5$, and $\geq 16 \mathrm{~cm} \times 2)$. For each of: lymphadenopathy, comb sign, abscesses and fistulae a score of 5 was added if present. The relationship between MRI score, calprotectin and HBI was evaluated using Kendall's rank correlation.

Results The mean MRI activity score was 15 (range 0-61.5) and was significantly correlated with calprotectin, Kendall's tau $b=0.42$, $p=0.0009$, but not with HBI, Kendall's tau $b=0.006, p=1$.

Conclusion Global Crohn's disease activity measured using a qualitative MRI score is correlated to the faecal calprotectin level. MRI is useful for the global assessment of Crohn's disease activity.

Competing interests None declared.

\section{PWE-232 ADAMDEC1: A NOVEL MOLECULE IN INFLAMMATION AND BOWEL DISEASE}

doi:10.1136/gutjnl-2012-302514d.232

${ }^{1} \mathrm{~N}$ R O'shea, ${ }^{* 1} \mathrm{~T}$ S Chew, ${ }^{1} \mathrm{G}$ Sewell, ${ }^{2} \mathrm{~S}$ Bloom, ${ }^{1} \mathrm{~A}$ Smith, ${ }^{1} \mathrm{~A}$ Segal. ${ }^{1}$ Department of Medicine, University College London, London, UK; ${ }^{2}$ Department of Gastroenterology, University College London, London, UK

Introduction Innate immunity is attenuated in patients with Crohn's disease (CD) with impaired neutrophil recruitment to skin and bowel, delayed clearance of Escherichia coli from the skin, and impaired secretion of pro-inflammatory cytokines from macrophages (Marks et al, 2006; Smith et al, 2009). The primary defect of acute inflammation results in failure to eradicate bacterial flora entering the bowel wall leading to the chronic granulomatous inflammation characteristic of CD. Microarray analysis of peripheral blood monocyte derived macrophage mRNA expression, confirmed by qPCR, revealed that ADAMDEC1 (ADAM like Decysin1) was under-expressed in $10 \%(6 / 60)$ of $C D$ patients. ADAMDEC1, a Metalloprotease and Decysin, is part of a family of proteins involved in wound healing and tissue repair, and is almost exclusively expressed in macrophages, dendritic cells and the gastrointestinal tract. To determine the role of this protein we examined $E$ coli induced inflammation and Dextran Sodium Sulphate (DSS) colitis in the Adamdec1 knockout (KO) mouse.

Methods In an acute colitis model, Adamdec1 $\mathrm{KO}$ mice were exposed to $2 \%$ DSS for 7 days. Controls, wild type (WT) litter mates, were age, weight and sex matched ( $n=11$ per group). Clinical colitis scores (weight loss, PR blood, loose stool) were recorded daily. Histology was obtained from small and large bowels. For bacterial inflammation, $5 \times 10^{8}$ heat killed $E$ coli ( $\mathrm{HkEc}$ ) were injected subcutaneously (SC) into two sites on the backs of KO and WT mice $(\mathrm{n}=8$ per group). Mice were weighed, injection sites inspected for ulceration and subcutaneous inflammatory nodules measured, daily. Injection sites were excised at different time points for histology and identification of infiltrating cells by FACS.

Results Adamdec1 KO mice were more susceptible to DSS colitis. They demonstrated higher clinical colitis scores with an earlier and more dramatic weight loss $(p<0.001)$. A more florid inflammatory response was seen on histology. In response to a subcutaneous injection of $E$ coli, Adamdec1 $\mathrm{KO}$ mice had significantly smaller inflammatory nodules and less ulceration at the injection sites after 48-72 h, compared with WT mice $(\mathrm{p}<0.001)$.

Conclusion Mice lacking Adamdec1 develop a phenotype that closely mirrors that observed in patients with $\mathrm{CD}$, an attenuated and delayed $E$ coli induced acute inflammatory and an increased susceptibility to bowel inflammation. These results suggest ADAMDEC1 plays an important role in the acute inflammatory response to bacteria and has a protective role within the intestine, reduced levels may have a pivotal role in the development and persistence of $\mathrm{CD}$

Competing interests None declared.

\section{PWE-233 SINGLE CENTRE COMPARISON OF MORTALITY, HOSPITAL RECORDED SERIOUS ADVERSE EVENTS (SAE) AND PRIMARY CARE RECORDED OPPORTUNISTIC INFECTIONS (OI) IN IBD PATIENTS TREATED WITH ANTI-TNF COMPARED TO THIOPURINES ALONE}

doi:10.1136/gutjnl-2012-302514d.233

${ }^{1} \mathrm{O}$ Waters, ${ }^{*}{ }^{1} \mathrm{M}$ Saunders, ${ }^{2} \mathrm{M}$ Bulsara, ${ }^{1} \mathrm{~T}$ Ahmad. ${ }^{1}$ Department of Gastroenterology, Royal Devon \& Exeter Hospital Foundation Trust, Exeter, UK; ${ }^{2}$ Department of Biostatistics, University of Notre Dame, Fremantle, Australia

Introduction The safety profile of anti-TNF therapy in IBD has been examined in previous case series, however these have been in tertiary centres, have not included a control cohort, or review of primary care records. We aimed to review SAE including primary care recorded OI, in patients treated with anti-TNF compared to a thiopurine alone.

Methods We studied two IBD cohorts: Cohort 1-All 212 anti-TNF treated patients between 1999 and 2010. Cohort 2-220 responders to an invitation sent to 365 patients treated with thiopurines only. A review of primary and secondary care records was conducted. A primary care OI was defined as any infection diagnosed or treated in primary care. Statistical analysis was performed using Stata V.12 
software. Proportional hazard regression model was used to assess mortality. Other SAE and OI were studied using logistic regression, t-test, and $\chi^{2}$ with Fishers exact.

Results Cohort 1: 137 (64.5\%) CD patients. 170 (80\%) received Infliximab (IFX) as their only anti-TNF treatment, 34 (16\%) IFX and Adalimumab (ADA) and 8 (4\%) ADA only. Cohort 2: 220 patients comprising 88 (40\%) CD. The mean age at drug initiation was 35.3 (SD 16.1) yrs in cohort 1, 45.7 (SD 15.9) yrs in cohort 2 ( $p<0.0001)$. Median follow-up after drug initiation in cohort 1 was 2.7 years (0-11.0), total 714.9 yrs, in cohort $26.4(0-30.8)$ yrs, total 1524 yrs. There were $8(4 \%)$ deaths in cohort 1 (1 cardiovascular (CVS), 3 sepsis, 3 solid organ malignancies, 1 haematological malignancy) and $5(2 \%)$ deaths in cohort 2 (2 CVS, 2 sepsis, 1 haematological malignancy). Cohort 1 patients had a higher mortality when corrected for age at diagnosis (HR 3.4 95\% CI 1.1 to 10.5) Abstract PWE-233 figure 1. In cohort 1 there were two cases of demyelination (1 suspected), 1 TB reactivation, 40 in-patient treated infections, 8 malignancies, seven cases of Clostridium difficile and no drug induced lupus. Hospital recorded SAE did not differ between cohorts 1 and 2 and no risk factors were identified. The mean number of primary care recorded OI was 5.8 (SD 5.8) in cohort 1 and 4.5 (SD 4.2) in cohort 2.

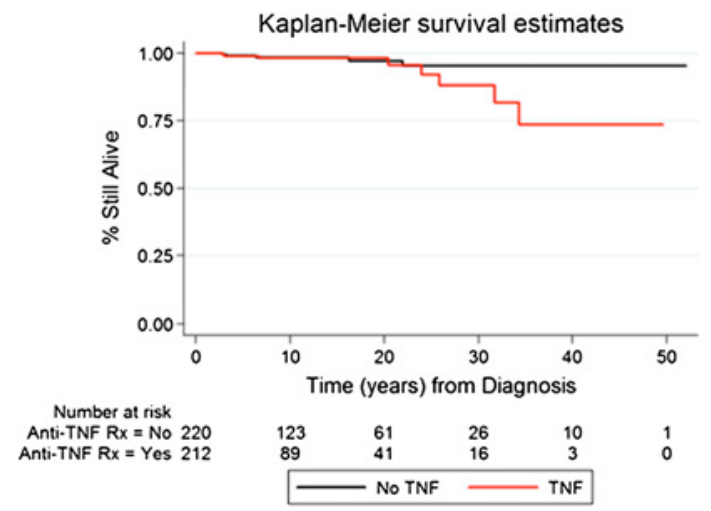

\section{Abstract PWE-233 Figure 1}

Conclusion The age at diagnosis adjusted mortality of IBD patients treat with anti-TNF is significantly greater than patients treated with thiopurines alone. Causality is unclear and may reflect underlying disease severity. Anti-TNF treated patients have similar rates of other SAE and primary care OI compared to patients treated with thiopurines alone.

Competing interests None declared.

\section{PWE-234 OPTIMISING THE RESPONSE TO THIOPURINE THERAPY: A SEARCH FOR NOVEL EXPLANATIONS FOR THIOPURINE HYPERMETHYLATION}

doi:10.1136/gutjnl-2012-302514d.234

${ }^{1} \mathrm{P}$ A Blaker, ${ }^{*}{ }^{2} \mathrm{~A}$ M Peters van Ton, ${ }^{2} \mathrm{M}$ Arenas Hernandez, ${ }^{1} \mathrm{M}$ A Smith, ${ }^{3} \mathrm{C}$ H Smith, ${ }^{1} \mathrm{P}$ Irving, ${ }^{2} \mathrm{~A}$ M Marinaki, ${ }^{1} \mathrm{~J} \mathrm{D}$ Sanderson. ${ }^{1}$ Department of Gastroenterology, Guy's \& St Thomas' Hospitals, London, UK; 'Purine Research Laboratory, Guy's \& St Thomas' Hospitals, London, UK; ${ }^{3}$ Department of Dermatology, Guy's \& St Thomas' Hospitals, London, UK

Introduction Thiopurines are not effective in up to $1 / 3$ of patients with inflammatory bowel disease (IBD) and $1 / 5$ have to discontinue therapy due to side effects. An important cause of these problems is thiopurine hypermethylation. This is a catabolic process leading to an unfavourable thiopurine metabolite profile (high methylmercaptopurine (MeMP) to low thioguanine nucleotide (TGN) ratio; >11:1), which cannot be predicted by measurement of thiopurine-S-methyltransferase (TPMT) activity. Importantly thiopurine hypermethylation can be circumvented with the use allopurinol in combination with a low dose thiopurine. The aim of this study is to establish the mechanism of thiopurine hypermethylation and identify predictive genetic markers to allow early combination therapy. We hypothesised that thiopurine hypermethylation occurs as a result of genetic factors that affect methylation flux and the cellular transport of methylated metabolites.

Methods 168 age and dose-matched patients prescribed AZA/6-MP were identified. Genomic DNA was extracted from EDTA blood samples of 76 patients demonstrating thiopurine hypermethylation and 92 patients with normal methylation profiles. Polymorphic sequence variants in genes predicted to affect thiopurine methylation flux and cellular metabolite transport were identified from single nucleotide polymorphism (SNP) databases and genotyped by Taqman assay. Associations were tested using Fisher's Exact test.

Results We found a significant association between the haplotype of rs9332377 $\mathrm{T}$ and rs4646316 C, which encodes a low-activity synonymous Catechol-O-methyltransferase (COMT) variant, and protection from thiopurine hypermethylation (rs9332377 T, $p=0.0178$, rs4646316 C, $p=0.03$ ). A polymorphism in the nucleobase transporter, $\mathrm{ABCB} 5$, was significantly associated with thiopurine hypermethylation ( $r 2031641 \mathrm{G} / \mathrm{G}, \mathrm{p}=0.0098$ ). The association was strengthened when patients with MeMP levels $>5000 \mathrm{pmol} / \mathrm{l}$ vs MeMP $<5000 \mathrm{pmol} / \mathrm{l}$ were compared ( $\mathrm{p}=0.0065$ ). Conclusion Changes in methylation flux due to the activity of methyltransferases other than TPMT affect the formation of thiopurine methylated metabolites, likely through direct competition for the essential co-factor S-adenosylmethionine. Furthermore, polymorphism in the ABCB5 gene, which affects the nucleotidebinding domain of this transporter, is associated with thiopurine hypermethylation, suggesting reduced cellular efflux of methylated metabolites. Further studies are now indicated to establish the role of these genetic markers in clinical practice.

Competing interests None declared.

\section{PWE-235 SCREENING USING THE EUROPEAN CROHN'S AND COLITIS ORGANISATION (ECCO) GUIDELINES DEMONSTRATES HIGH STRONGYLOIDES SERO- PREVALENCE IN MIGRANTS WITH INFLAMMATORY BOWEL DISEASE}

doi:10.1136/gutjnl-2012-302514d.235

${ }^{1} \mathrm{P}$ J Smith, ${ }^{* 1} \mathrm{~B}$ Theis, ${ }^{1} \mathrm{~N}$ R O'Shea, ${ }^{1} \mathrm{R}$ Vega, ${ }^{1} \mathrm{~S}$ McCartney, ${ }^{1,2} \mathrm{M}$ Brown, ${ }^{1} \mathrm{~S}$ L Bloom. ${ }^{1}$ Department of Gastroenterology, University College London Hospital, London, UK ${ }^{2}$ Hospital for Tropical Diseases, London, UK

Introduction Strongyloidiasis can persist and cause hyperinfection years after acquisition when host immunity is impaired. European Crohn's and Colitis Organisation guidelines ${ }^{1}$ on opportunisitc infections recommend that Inflammatory Bowel Disease (IBD) patients returning from endemic areas be screened. However, prevalence of intestinal helminths in migrant IBD patients is unknown. We investigated the sero-prevalence of Strongyloidiasis and factors associated with infection.

Methods Migrant patients attending IBD clinic over a 10 -month period, with a diagnosis of Crohn's disease (CD) or Ulcerative colitis (UC), were tested for Strongyloides serology. Eosinophil count and inflammatory markers were measured. Ethnicity was used as a proxy for migrant status. Sero-positive patients were followed-up with a Strongyloides charcoal culture before treatment with Ivermectin. Repeat eosinophil count and inflammatory markers were performed 3 months later. $T$ test and $\chi^{2}$ analysis $(p<0.05)$ were performed using SPSS for Windows.

Results 97 migrant patients (54 CD vs 43 UC) were tested. 13/97 patients were sero-positive. In both groups, over $70 \%$ of patients were from Asia. Mean eosinophil counts $\left(\times 10^{9} / 1\right)$ were not different between the two groups $(0.29$ vs $0.22, \mathrm{p}>0.05)$. No significant 